\title{
Target-Dependent Feedforward Inhibition Mediated by Short-Term Synaptic Plasticity in the Cerebellum
}

\author{
Jin Bao, ${ }^{1}$ Kerstin Reim, ${ }^{2}$ and Takeshi Sakaba ${ }^{1}$ \\ ${ }^{1}$ Independent Junior Research Group Biophysics of Synaptic Transmission, Max Planck Institute for Biophysical Chemistry, D-37077 Göttingen, Germany, \\ and ${ }^{2}$ Department of Molecular Neurobiology and Center for Molecular Physiology of the Brain, Max Planck Institute of Experimental Medicine, D-37075 \\ Göttingen, Germany
}

Cerebellar feedforward inhibition (FFI) is mediated by two distinct pathways targeting different subcellular compartments of Purkinje cells (PCs). The axon of the granule cell, the parallel fiber, makes excitatory synapses not only onto PCs but also onto two types of interneurons, basket and stellate cells. Basket and stellate cells then send inhibitory signals to the soma and dendrites of Purkinje cells, respectively. Functional differences between somatic and dendritic FFI pathways, however, remain unknown. Here we address this question by examining how basket and stellate cells are recruited dynamically under high-frequency granule cell inputs at mice cerebellum. Short-term plasticity of various synapses within the FFI circuit has been explored. Unexpectedly, the parallel fiber synapse, which was considered to be facilitating during repetitive stimulation, shows depression, when the postsynaptic target is a basket cell. Other factors in the FFI circuit, such as firing properties of interneurons and dynamics of inhibitory synapses, are similar between somatic and dendritic pathways. The target-dependent parallel fiber synaptic plasticity has functional consequences for the two FFI pathways, because we observe that PCs receive transient somatic inhibition during $50 \mathrm{~Hz}$ stimulation of granule cells but persistent dendritic inhibition.

\section{Introduction}

In the cerebellum, the two excitatory pathways to the Purkinje cell (PC), climbing and parallel fibers (PFs), have different properties. Climbing fibers elicit large postsynaptic responses, which are depressed during repetitive stimulations (Eccles et al., 1966c; Silver et al., 1998), whereas PFs elicit small postsynaptic responses (Barbour, 1993), which are facilitated strongly during high-frequency activity (Atluri and Regehr, 1996). Parallel fibers, the axons of granule cells (GCs), also make synapses onto two types of interneuron, basket (BC) and stellate (SC) cells, which in turn provide inhibitory inputs to the soma and dendrites of Purkinje cells, respectively (Eccles et al., 1966b; Palay and ChanPalay, 1974) (see Fig. 1A). This results in feedforward inhibition (FFI), which can rapidly terminate the membrane depolarization of PCs induced by PF inputs (Eccles et al., 1967; Brunel et al., 2004; Mittmann et al., 2005). FFI has an important role in the cerebellar function in vivo (Miyashita and Nagao, 1984; Eccles et al., 1966d; Wulff et al., 2009), but it is not known how inhibitory neurons are recruited dynamically. Specifically, it is not known whether the two types of interneurons are recruited differently during high-frequency GC inputs (Jörntell and Ekerot, 2006). Although BCs and SCs share some morphological and physiological features (Vincent and Marty, 1996; Sultan and Bower, 1998)

\footnotetext{
Received Jan. 18, 2010; revised April 26, 2010; accepted May 7, 2010.

We thank A. Ohle at the Max Planck Institute of Experimental Medicine for invaluable help with animal husbandry. We also thank Erwin Neher and Gerard Borst for helpful suggestions on this study.

Correspondence should be addressed to Jin Bao or Takeshi Sakaba, Independent Junior Research Group Biophysics of Synaptic Transmission, Max Planck Institute for Biophysical Chemistry, D-37077 Göttingen, Germany. E-mail: jbao@gwdg.de or tsakaba@gwdg.de.

DOI:10.1523/JNEUROSCI.0276-10.2010

Copyright $\odot 2010$ the authors $\quad 0270-6474 / 10 / 308171-09 \$ 15.00 / 0$
}

and protein expression (Kosaka et al., 1993; Caillard et al., 2000), they have distinct axonal structures. Basket cells have a large terminal innervating the soma of the Purkinje cells. Besides the target difference, it remains possible that both input and output synaptic dynamics during repetitive activity might differ between BCs and SCs, as has been observed in different types of interneurons in hippocampus (Pouille and Scanziani, 2004) and neocortex (Beierlein et al., 2003). A difference in firing properties or synaptic dynamics of BCs and SCs would indicate that somatic and dendritic inhibitions onto PCs are differentially regulated. Here, we studied how basket and stellate cells were recruited in the FFI circuit from mouse cerebellar slices. We found that inhibitory synapses formed by these two cell types on PCs had similar short-term dynamics except that synaptic strengths were different by sevenfold. Firing properties of the two types of interneurons were also similar. Surprisingly, the granule cell $\rightarrow$ basket cell synapse had distinct dynamics, exhibiting short-term depression during $50 \mathrm{~Hz}$ excitation. As a result of the target-dependent synaptic plasticity of the granule cell synapse, somatic and dendritic inhibition are rendered with different temporal characteristics to transform the information carried by granule cell spikes to Purkinje cells.

\section{Materials and Methods}

Slice preparation. C57BL/6N male and female mice (postnatal days 1628) were decapitated in accordance with the guidelines of the German law on animal protection. Coronal or parasagittal brain slices $(250 \mu \mathrm{m})$ of cerebellar vermis were obtained with a Leica VT1000S slicer. During slicing, the cerebellum was kept in ice-cold solution containing the following (in mM): $60 \mathrm{NaCl}, 120$ sucrose, $25 \mathrm{NaHCO}_{3}, 1.25 \mathrm{NaH}_{2} \mathrm{PO}_{4}, 2.5$ $\mathrm{KCl}, 25 \mathrm{D}$-glucose, $0.1 \mathrm{CaCl}_{2}$, and $3 \mathrm{MgCl}_{2}$. Slices were incubated at $35^{\circ} \mathrm{C}$ for $1 \mathrm{~h}$ in a saline solution $(305 \mathrm{mmol} / \mathrm{kg}$ ) containing the following (in 
mM): $125 \mathrm{NaCl}, 2.5 \mathrm{KCl}, 25 \mathrm{NaHCO}_{3}, 1.25$ $\mathrm{NaH}_{2} \mathrm{PO}_{4}, 2 \mathrm{CaCl}_{2}$, and $1 \mathrm{MgCl}_{2}, \mathrm{pH} 7.3-7.4$ with continuous bubbling $\left(95 \% \mathrm{O}_{2}\right.$ and $5 \%$ $\mathrm{CO}_{2}$ ). Recordings were performed at $33-34^{\circ} \mathrm{C}$ with a chamber heater and an inline solution heater (Warner Instruments). Heated saline solution was perfused to the recording chamber at $2-3 \mathrm{ml} / \mathrm{min}$.

Electrophysiology. Slices were visualized with differential interference contrast optics and a $60 \times$ water-immersion objective (Olympus). Extracellular stimulation was performed by inserting the tip of a glass pipette filled with saline solution into the granule cell layer. BCs and SCs were distinguished according to their location in the molecular layer (Palay and ChanPalay, 1974). BCs are located close to the Purkinje cell soma; SCs are located between the middle of the molecular layer and the pia. Distinction between the two types of interneurons was confirmed by dialyzing Alexa 488 to visualize the morphology, as described in a previous study (Sakaba, 2008). BCs were identified not only by large axon terminals but also their bigger inhibitory synaptic strength onto PCs (see Fig. 5C). Whole-cell patch-clamp recordings were obtained using an EPC10/2 amplifier controlled by the Windows program patch master (Heka). Glass pipettes were 3-4 $\mathrm{M} \Omega$ for PCs and 5-6 $\mathrm{M} \Omega$ for BCs and SCs. For voltage-clamp experiments, the internal solution contained the following (in mM): 135 Csgluconate, $5 \mathrm{CsCl}, 5 \mathrm{QX}-314$ (chloride salt) $N$-(2,6-dimethylphenylcarbamoylmethyl)triethylammonium (Tocris), 10 HEPES, 5 MgATP, 0.5 NaGTP, and 1 EGTA, pH 7.2 (310 mOsm). The junction potential was estimated to be $10 \mathrm{mV}$ from the reversal potential of the AMPA receptor current triggered by an excitatory synaptic input. The reported voltage here were not corrected for the junction potential. EPSCs were recorded in the presence of the selective $\mathrm{GABA}_{\mathrm{A}}$ receptor antagonist SR 95531 [2-(3-carboxypropyl)-3-amino-6(4-methoxyphenyl)pyridazinium bromide] (Sigma) at a holding potential of $-65 \mathrm{mV}$. Presynaptic and postsynaptic simultaneous recordings of $\mathrm{BC}(\mathrm{SC}) \rightarrow$ PC pairs were performed with a high $\left[\mathrm{Cl}^{-}\right]$internal solution for PCs, containing $135 \mathrm{~mm} \mathrm{CsCl}$ instead of $135 \mathrm{~mm}$ Cs-gluconate, which magnified IPSCs at a holding potential of $-70 \mathrm{mV}$. If not stated, intracellular $\left[\mathrm{Cl}^{-}\right.$] was $10 \mathrm{~mm} . R_{\mathrm{s}}$ compensation was performed by EPC10/2 amplifier, and parameters were selected manually. For PCs, $>60 \%$ of $R_{\mathrm{s}}$ was compensated, leaving $<4 \mathrm{M} \Omega$ uncompensated series resistance. For $\mathrm{BCs}$ and $\mathrm{SCs}$, the uncompensated $R_{\mathrm{s}}$ was $<10 \mathrm{M} \Omega$. Current-clamp experiments were performed with an internal solution containing the following (in $\mathrm{mm}$ ): $135 \mathrm{~K}$-gluconate, $10 \mathrm{KCl}, 10 \mathrm{HEPES}, 5 \mathrm{MgATP}, 0.5$ $\mathrm{NaGTP}$, and 0.1 EGTA. For coronal slices, stimulation pipettes were placed 100-200 $\mu \mathrm{m}$ from the recorded cell soma along the direction of the parallel fiber. In parasagittal slices, parallel fibers ran perpendicular to the slice surface; therefore, stimulation pipettes were placed relatively close to the recorded cells. Stimulation intensity was adjusted to elicit small but reliable postsynaptic responses.

Data acquisition and analysis. Data were filtered at $5-10 \mathrm{kHz}$ and sampled at $50 \mathrm{kHz}$. Offline analysis was performed by custom-written programs using Igor Pro (Wavemetrics) and Matlab (MathWorks). Sweeps were repeated 10-20 times in the train stimulation experiment and were averaged within a given cell. Peak amplitudes were detected as a local minimum (or maximum) for each response. In the case of a slow decay signal from the previous response superimposed with the current one, fitting of the previous decay to an exponential function was performed to offset the current response to retrieve the evolution of synaptic current activity. Unpaired $t$ test was performed with $\alpha=0.05$.

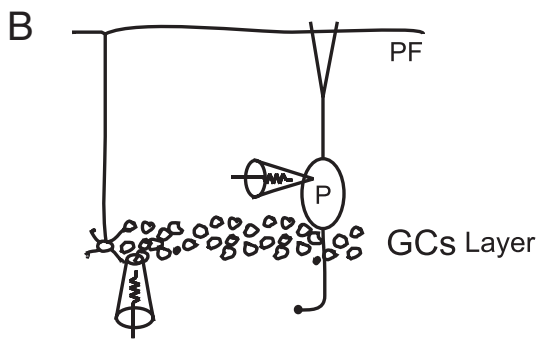

D $50 \mathrm{~Hz}$ Granule cell stimulation

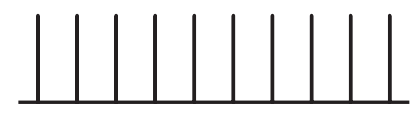

post-synaptic currents of Purkinje Cell

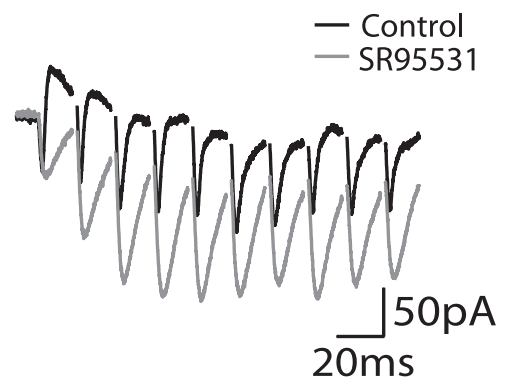

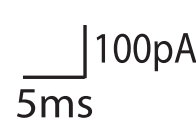

Figure 1. FFI circuits activated by granule cell stimulation. $A$, An illustration of somatic and dendritic FFI by $\mathrm{PF} \rightarrow \mathrm{BC} \rightarrow \mathrm{PC}$ recording. $C$, A single extracellular stimulation applied to the granule cell layer elicited a PSC recorded from the Purkinje cell soma. of 10 sweeps, and stimulus artifacts are removed from PSC traces. $D$, Trains of stimulation pulses were applied to the granule cell layer. An example of a $50 \mathrm{~Hz}$ train and its responses are shown in a similar way to $C$. B, Basket cell; P, Purkinje cell; S, Stellate cell.

\section{Results}

\section{Feedforward inhibition circuits activated by granule cell stimulation}

To activate FFI circuit, we stimulated GCs (Fig. 1B) rather than PFs in the molecular layer to avoid direct stimulations of interneurons and the recruitment of fibers during repetitive stimulations (Barbour, 1993; Marcaggi and Attwell, 2005; Beierlein et al., 2007). The stimulation strength was adjusted such that the amplitudes of postsynaptic responses were reliable and comparable with the ones in the previous studies (Mittmann et al., 2005). The soma of the PC was voltage clamped. Stimulations of GCs activated feedforward inhibitory currents in the PC (Fig. 1C,D), as has been shown in previous studies (Barbour, 1993; Mittmann et al., 2005). An excitatory inward current was followed by an outward current, which was blocked by the $\mathrm{GABA}_{\mathrm{A}}$ receptor antagonist SR 95531, indicating a delayed disynaptic inhibition. PFs form glutamatergic synapses on the dendrites of PCs, BCs, and SCs. BCs and SCs are GABAergic interneurons that innervate the soma and the dendrites of Purkinje cells, respectively (Fig. 1A). Therefore, FFIs consist of two pathways mediating somatic and dendritic inhibition. During a $50 \mathrm{~Hz}$ train of GC stimulation (Fig. $1 D$ ), both monosynaptic excitatory currents and disynaptic inhibitory currents observed at the PC soma showed short-term plasticity, implying that FFIs were dynamically recruited during activity. The underlying mechanism and functional implication of excitatory and inhibitory short-term synaptic plasticity in the FFI circuit will be analyzed in the following sections. 

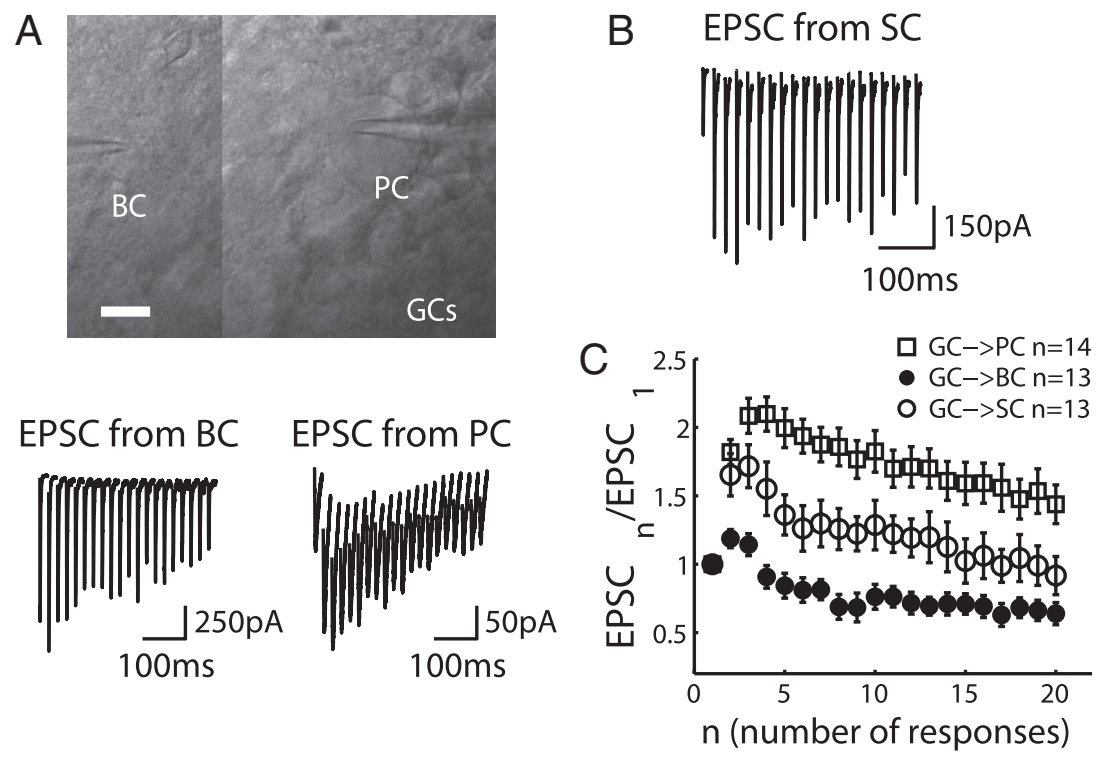

Figure 2. Target-dependent plasticity of granule cell synapses. A, Imaging of simultaneous patch-clamp recording from Purkinje cell and basket cell (scale bar, $10 \mu \mathrm{m}$ ). EPSCs recorded from the BC during $50 \mathrm{~Hz} \mathrm{GC}$ stimulations are depressing, and those recorded from the $P C$ are facilitating. Cells were voltage clamped at $-65 \mathrm{mV}$ and in the presence of SR 95531. Each trace is an average of 10 sweeps. $\boldsymbol{B}$, EPSC of an SC under the same experiment condition as in $\boldsymbol{A}$. $\boldsymbol{C}$, Peak amplitudes of EPSCs recorded during the train are normalized to the first one and plotted against the number of responses. Each trace represents an average from $14 \mathrm{PCS}$ (open squares), 13 BCs (filled circles), and 13 SCs (open circles) (mean \pm SEM).
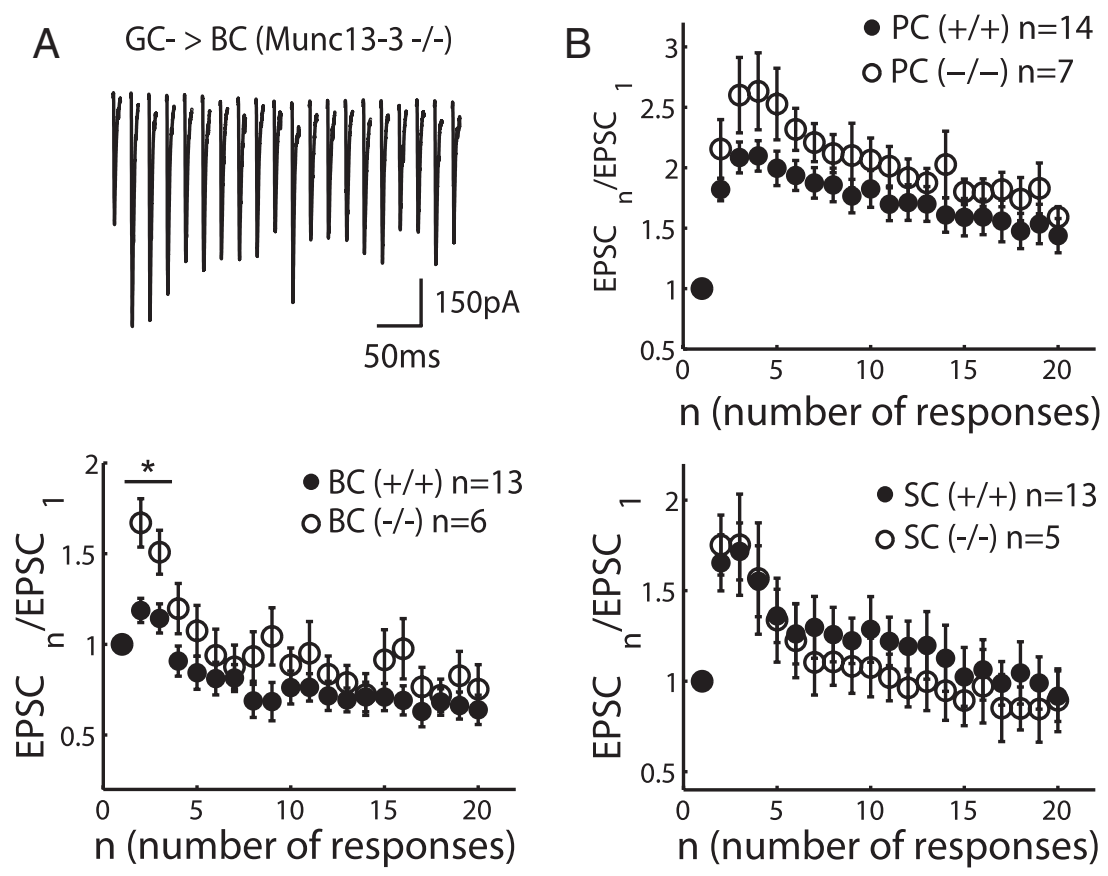

Figure 3. Deletion of Munc13-3 enhances the paired-pulse facilitation at $\mathrm{GC} \rightarrow \mathrm{BC}$ synapse, but $\mathrm{GC} \rightarrow \mathrm{PC}$ and $\mathrm{GC} \rightarrow \mathrm{SC}$ synapses are unaltered. $\boldsymbol{A}$, An example trace of EPSCs recorded from a basket cell of Munc13-3 K0 mice (top) shows no depression. The cell was voltage clamped at $-65 \mathrm{mV}$ in the presence of SR 95531, and $50 \mathrm{~Hz}$ trains of stimulation were applied to the granule cell layer. Normalized EPSCs were averaged from six recordings in $\mathrm{KO}$ mice (open circles in bottom) present a significant increase of the paired-pulse ratio compared with their wild-type littermates (filled circles). ${ }^{*} p<0.01$. $\boldsymbol{B}$, The same as $\boldsymbol{A}$, but the postsynaptic cells were Purkinje cells and stellate cells.

Target-dependent short-term plasticity of parallel fiber synapses

During repetitive stimulation, synapses can exhibit different forms of short-term plasticity (Zucker and Regehr, 2002). To investigate the consequence of short-term synaptic plasticity on the FFI circuit function, we first examined the short-term plasticity at different PF synapses formed by the GC axon terminals with PCs, BCs, and SCs. To preserve PFs, experiments were performed on coronal slices. Trains of extracellular stimulation were applied to GCs with regular interstimulus intervals in the presence of a $\mathrm{GABA}_{\mathrm{A}}$ receptor blocker. The evoked EPSCs were recorded from the target cells, and 10-20 sweeps were averaged (Fig. $2 A, B)$. Some experiments were performed with simultaneous voltage-clamp recordings from $\mathrm{PC}$ and $\mathrm{BCs}$, as shown in Figure $2 A$ (top). EPSCs recorded from the PCs showed strong facilitation during 50 $\mathrm{Hz}$ train stimulation (Fig. 2A, bottom, right), consistent with previous studies (Barbour, 1993; Dittman et al., 2000; Isope and Barbour, 2002). The EPSC sizes were also comparable with the previous studies. In contrast, EPSCs recorded from the BCs showed minor paired-pulse facilitation, followed by a pronounced depression under the same stimulation condition (Fig. $2 A$, bottom, left). EPSCs recorded at another type of interneuron, SCs, showed strong facilitation, similar to GC $\rightarrow$ PC synapses (Fig. 2 B). In Figure $2 C$, the peak amplitudes of EPSCs were divided by the first one in the train and plotted against the stimulus number (mean \pm SEM), showing that PF synapses exhibited targetdependent short-term plasticity: GC $\rightarrow$ PC and GC $\rightarrow$ SC synapses are facilitating synapses, whereas GC $\rightarrow$ BC synapses are depressing synapses [comparing PCs $(n=14)$ and BCs $(n=13), p<0.0001$; comparing SCs $(n=13)$ and BCs $(n=13), p<0.05]$. Target-dependent synaptic plasticity is known to be mainly mediated by presynaptic mechanisms, such as differences in release probability among synapses (Reyes et al., 1998; Lawrence and McBain, 2003; Beierlein et al., 2007). To gain additional insights of the difference between GC $\rightarrow$ BC and GC $\rightarrow$ SC synapses, we studied knockout (KO) mice of Munc13-3, a presynaptic protein implicated in synaptic vesicle priming (Augustin et al., 1999; Basu et al., 2007). Deletion of Munc13-3, a cerebellar-specific isoform of Munc13 (Augustin et al., 2001), has been shown to induce an increase of paired-pulse facilitation in granule cell $\rightarrow$ Golgi cell synapses (Beierlein et al., 2007). We observed an increase of the pairedpulse facilitation also in $\mathrm{GC} \rightarrow \mathrm{BC}$ synapse (Fig. $3 A$ ) but no significant changes in $\mathrm{GC} \rightarrow \mathrm{PC}$ and GC $\rightarrow$ SC synapses (Fig. $3 B$ ). The deletion of Munc13-3 makes the GC $\rightarrow$ BC synapses more facilitating, like $\mathrm{GC} \rightarrow \mathrm{SC}$ synapses. In other words, the presynaptic mechanism is important for target-dependent synaptic plasticity of the PF synapses. Inhibitory synapses were not affected by deletion of 
Munc13-3 ( $n=3$; data not shown). Localized phenotype of Munc13-3 in GC $\rightarrow$ $\mathrm{BC}$ synapses will be used for examining the roles of somatic inhibition in a later section (see Fig. 8).

Target-dependent short-term synaptic plasticity results in distinct spike output patterns at the two types of interneurons

The difference in short-term plasticity of the input synapse to the two interneurons-short-term facilitation in GC $\rightarrow$ SC synapse and depression in GC $\rightarrow$ BC synapse-prompted us to further examine its impact on their spike output. First, we injected a $500 \mathrm{~ms}$ current pulse in the soma under current-clamp mode to examine whether BCs and SCs exhibited a similar firing pattern. BCs and SCs fired regularly without adaptation during constant current injection ( $n=5$ cells for BCs; $n=8$ cells for SCs); an example is shown in Figure $4 A$. Their firing pattern can be classified as fast spiking, which is commonly observed in inhibitory interneurons (Markram et al., 2004; Somogyi and Klausberger, 2005). The number of spikes was plotted against the amplitudes of injected current (shown in Fig. $4 A$, bottom), indicating that the spiking property of BCs and SCs were similar. We then applied $50 \mathrm{~Hz}$ GC stimulation and recorded the membrane potential from the BCs and SCs. The experiment was performed without SR 95531 to reveal firing patterns under physiological conditions. Spiking events were plotted as a raster plot, and the probability of spiking was calculated by taking the ratio between the number of evoked spikes across all the sweeps for each stimulation in the train and the total number of repeated sweeps (Fig. $4 B$, one example from each cell type). For BCs, the spiking probability decreased after the onset of the stimulation. The average spiking probability at the onset of the stimulation is $88 \pm 4.4 \%$ (Fig. $4 \mathrm{~B}$, bottom), and it decreases to $50 \pm 1.5 \% 200 \mathrm{~ms}$ after the onset of the stimulation. Thus, a high-frequency spike input from GCs (Chadderton et al., 2004; Jörntell and Ekerot, 2006) would be converted to the transient spike outputs of BCs. On average, SCs had a much lower initial spiking probability that was $48 \pm 7.7 \%$. The probability increased to $77 \pm 5.5 \%$ at the third response and stayed at this level within $400 \mathrm{~ms}(p<0.01)$. Therefore, GCs trigger spikes in SCs more persistently than in BCs under a highfrequency train of stimulation.

\section{Inhibitory synapses are depressing}

It is possible that signals coming to the interneurons are further filtered by dynamics of inhibitory synapses before reaching PCs. Different types of interneurons exhibit distinct short-term synaptic plasticity in neocortex and hippocampus (Beierlein et al., 2003; Hefft and Jonas, 2005), but a systematic comparison has not been made in the cerebellum. We therefore examined the dynamics of $\mathrm{BC} \rightarrow \mathrm{PC}$ and $\mathrm{SC} \rightarrow$ PC synapses by simultaneous
B $50 \mathrm{~Hz}$ synaptic stimulation
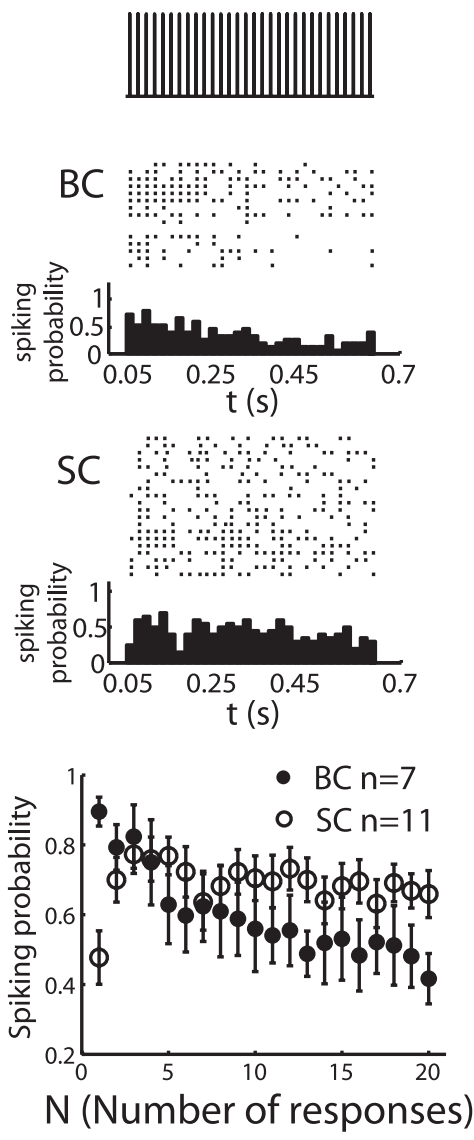
$\downarrow 100 \mathrm{pA}$ $100 \mathrm{~ms}$

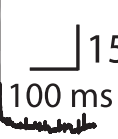

\section{$5 \mathrm{mV}$} $15 \mathrm{mV}$ $100 \mathrm{~ms}$

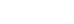

Figure 4. Spiking of interneurons in FFI circuit. $A, A 200 \mathrm{pA}$ current injection to the soma of $\mathrm{BC}$ and $\mathrm{SCs}$ induced $140 \mathrm{~Hz}$ regular inst the amplitudes of injected currents (bottom). B, Spikes were detected from BCs and SCs and plotted as a raster plot during a $50 \mathrm{~Hz}$ train of granule cell stimulation. Spikes were counted from a $5 \mathrm{~ms}$ time window after each stimulation pulse. Spiking probabilities are plotted beneath the raster plot. Summarized spiking probability is shown for both cell types (bottom).

patch-clamp recordings from a connected pair of cells. When a train of short current pulses was injected into the interneuron, spikes were elicited faithfully (Sakaba, 2008). The evoked IPSCs were recorded from the $\mathrm{PC}$ using a pipette solution with elevated intracellular $\left[\mathrm{Cl}^{-}\right]$. Figure $5 \mathrm{~A}$ shows averaged inhibitory current traces from 10 to 20 sweeps in response to a $50 \mathrm{~Hz}$ spike train. Both $\mathrm{BC} \rightarrow \mathrm{PC}$ and $\mathrm{SC} \rightarrow \mathrm{PC}$ synapses are depressed ( $n=6$ pairs of BC-PC; $n=4$ pairs of SC-PC). The normalized IPSCs demonstrate that both synapses have similar short-term synaptic dynamics (Fig. 5B). However, we observed a sevenfold difference in their absolute inhibitory strengths $(\mathrm{BC} \rightarrow \mathrm{PC}, 1.73 \pm 0.55 \mathrm{nA}$; $\mathrm{SC} \rightarrow \mathrm{PC}, 0.24 \pm 0.04 \mathrm{nA}$ ), which would be mostly attributable to the different size of the presynaptic terminals (Palay and ChanPalay, 1974). Munc13-3 KO mice did not change the depression time course at the $\mathrm{BC} \rightarrow$ PC synapse ( $n=3$; data not shown), confirming that Munc13-3 was not involved in inhibitory synaptic transmission (Augustin et al., 2001).

\section{Persistent dendritic inhibition evoked by high-frequency granule cell input}

We have shown that dendritic FFI is mediated through a facilitating excitatory synapse and a depressing inhibitory synapse. We expected that the balance between the facilitating excitatory synapse and the depressing inhibitory synapse would be achieved at a certain input frequency and thus persistent dendritic inhibition 
A current injection to interneuron
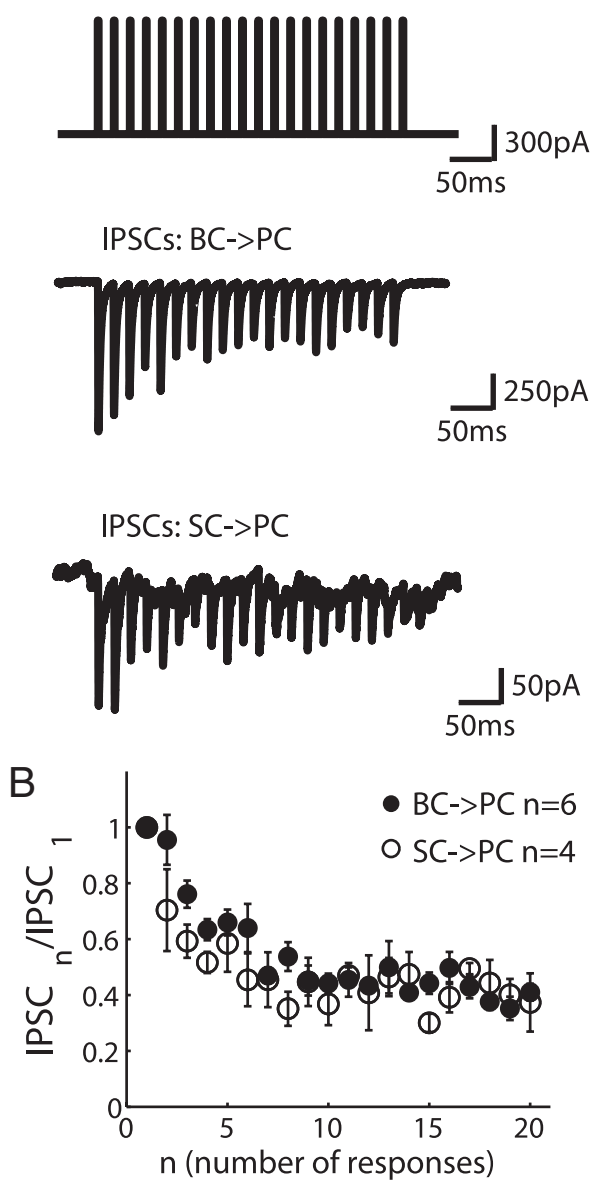

Figure 5. Depressing inhibitory synapses in FFI circuit. $A, A 50 \mathrm{~Hz}$ current pulse injection to a BC or SC elicited a reliable spike during each pulse (spikes were not shown). Simultaneous voltage-clamp recording with $135 \mathrm{~mm}$ intracellular $\left[\mathrm{Cl}^{-}\right]$from a $\mathrm{PC}$ that was innervated by the interneuron shows inward inhibitory current (negative) with depressing dynamics. Holding potential was $-70 \mathrm{mV}$. $\boldsymbol{B}$, Normalized IPSCs are plotted against the number of responses showing similar dynamics of these two synapses (mean \pm SEM).

could be observed as a result. To test this specifically, we applied spike patterns obtained in Figure $4 B$ (the spike pattern of one example stellate cell recorded by stimulating GCs) into a single stellate cell and examined the resulting short-term plasticity of IPSCs recorded at the PC, which was innervated by the SC. The $\mathrm{PC}$ was voltage clamped with a patch pipette containing cesium and high $\left[\mathrm{Cl}^{-}\right]$. Figure $6 \mathrm{~A}$ shows the average IPSCs obtained from "realistic" spike patterns. In contrast to a regular spike train (Fig. 5), the average IPSCs showed facilitation. The data are summarized from seven cells in Figure $6 B$, which shows no sign of synaptic depression during this stimulation protocol. It is likely that a relatively high failure rate of spiking is the main cause for synaptic facilitation seen in Figure 6, demonstrating that targetdependent synaptic plasticity of the GC synapse has an important consequence. The realistic spike pattern of SCs thus constitutes one mechanism that balances facilitating input synapse and depressing output synapse. As shown in Figure 4, failure rates of spiking in response to granule cells varied among cells. Therefore, it is possible that inhibitory synapses may show less facilitation when the firing rates are higher at the stellate cells, and, as a result, overall dendritic inhibition is less facilitative than the one seen in Figure 6.

Next, to examine whether inhibitory synaptic inputs were persistent at the dendrite, we performed dendritic patch-clamp re- cordings to isolate dendritic inhibition from somatic inhibition. Dendritic patch clamp was performed on the dendritic arbor of a PC, $>100 \mu \mathrm{m}$ from the soma. A $50 \mathrm{~Hz}$ GC stimulation protocol was the same as in Figure 2. Dendritic membrane potentials were recorded from the dendritic patch pipette shown as the black trace in Figure $7 A$ (top). When the inhibitory synaptic inputs were blocked by SR 95531, we recorded the EPSPs shown as the gray trace under the same stimulation condition as control. Each trace is an average of five sweeps. GC stimulation pipette was placed on the side of the recording dendritic branch, and the intensity was adjusted to minimum the recruitment of somatic activation. In the presence of inhibition, the dendritic depolarization during the train was limited. The decay of each response was also accelerated. Each individual PSP in the train was offset by the decay of its previous response (Fig. $7 \mathrm{~A}$, bottom) to retrieve the evolution of membrane potential attributable to the slow decay of synaptic current. Contribution of IPSPs was then calculated by subtracting the two traces (Fig. $7 B$, top). The normalized peak amplitudes of IPSPs were plotted in Figure $7 B$ (bottom, an average from three cells), showing a slight depression ( $80 \pm 4.7 \%$ steady-state depression averaged from the last 10 responses). Compared with the direct stimulation of an SC in Figure $5 B$ (42 $\pm 1.9 \%$ steady-state depression), inhibition depressed much less when GCs were stimulated. In the experiment of Figure 7, inhibitory conductance was not directly measured because of the difficulty of dendritic voltage clamp. Using current clamp does not necessarily measure the inhibitory synaptic conductance directly because of confounding effects, such as activation of voltage-gated channels and change in the driving force of channels. Because voltage changes were relatively small in our experiments, together with Figure 6, it is likely that dendritic inhibition is recruited persistently under high-frequency GC inputs $(50 \mathrm{~Hz}$ for 20 times) as a result of the counterbalance between the facilitating excitatory and depressing inhibitory synapse. Note that other factors may contribute to persistent inhibition: most likely, multiple SCs contribute to dendritic inhibitions and SCs do not always fire in response to the GC stimulation (Figs. 4, 6). Combination of these two factors may further prevent depression of $\mathrm{SC} \rightarrow$ PC synapses. Compared with Figure 6, dendritic inhibition is less facilitative in Figure 7. This is most likely because the initial spiking rates are variable among stellate cells, and some synapses show less facilitation or even depression when the initial spiking rate is high. As a result, overall dendritic inhibition may become less facilitative.

\section{Phasic somatic inhibition during high-frequency activity}

Somatic inhibition of Purkinje cell is mediated by large basket cell inputs. The disynaptic IPSCs measured from a PC soma in response to GC stimulation are the summation of somatic and dendritic inhibition, which can be estimated by subtracting the synaptic currents with and without SR 95531 (Fig. 8A). We observed that IPSCs were depressed to $48 \pm 1.5 \%$ during $50 \mathrm{~Hz}$ GC stimulation. Because dendritic inhibition did not show such strong depression (Figs. 6, 7), the depression of disynaptic IPSCs seen in Figure $8 \mathrm{~A}$ must be determined by the $\mathrm{GC} \rightarrow \mathrm{BC} \rightarrow \mathrm{PC}$ pathway. Because deletion of Munc13-3 converted GC $\rightarrow$ BC synapse from depression to facilitation, one might expect that disynaptic IPSCs would be less depressed during a train stimulation in Munc13-3 KO mice. Indeed, as shown in Figure $8 \mathrm{~B}$, somatic disynaptic IPSCs showed facilitation lasting $>100 \mathrm{~ms}$ after the onset of $50 \mathrm{~Hz}$ stimulation in $\mathrm{KO}$ mice. It is important to note that the synaptic connections other than GC $\rightarrow$ BC synapse are unchanged in Munc13-3 KO mice (see above). 
To further prove that $\mathrm{BC}$ inputs mediated phasic somatic inhibition, we performed simultaneous patch-clamp recordings on a pair of connected BCs and PCs as in Figure 9. When the BC was in current-clamp mode (control condition), it fired when the excitatory inputs from GCs exceeded the spiking threshold. The synaptic current recording from the $\mathrm{PC}$ is shown as a black trace in Figure 9; it consists of monosynaptic excitatory currents and feedforward inhibitory currents. When the $\mathrm{BC}$ was voltage clamped at $-70 \mathrm{mV}$ to prevent firing, we considered this $\mathrm{BC}$ to be silenced (Fig. 9, gray). Each trace is an average of 10-20 sweeps in the two conditions. Subtraction of these two traces resulted in the inhibitory current from the recorded basket cell to the Purkinje cell, which showed substantial depression within tens of milliseconds. An average of $40 \pm 5.8 \%$ steady-state depression was estimated from four cells. The time course of single BC inhibition was similar to the macroscopic disynaptic IPSCs (Fig. $8 \mathrm{~A}$ ), suggesting that a majority of somatic IPSCs were contributed by basket cells. We conclude that depressing somatic inhibition is a consequence of $\mathrm{GC} \rightarrow \mathrm{BC}$ and $\mathrm{BC} \rightarrow$ PC synapses, both of which are depressing.

\section{Discussion}

The present work demonstrates that two feedforward inhibitory circuits in cerebellar vermis have distinct filtering properties (Abbott and Regehr, 2004), and this difference is mediated mainly by the targetdependent short-term synaptic plasticity of the PF synapses. In the somatic inhibition pathway, depressing PF synapses in series with depressing BC synapses result in phasic inhibition onto the soma of a PC. In the dendritic inhibition pathway, facilitating PF synapses balance depressing SC synapses to allow more persistent inhibition onto the dendrite of a PC compared with somatic inhibition. Strong somatic inhibition works reliably within low-frequency ranges to inhibit spike output of PCs (Mittmann and Häusser, 2007). Persistent dendritic inhibition can operate at higher frequencies to counteract the local dendritic excitatory inputs from PF synapses.

\section{Mechanisms of target-differential dynamics}

The PF synapse has always been considered as a facilitating synapse (Konnerth et al., 1990; Dittman et al., 2000; Beierlein et al., 2007), whereas it has been reported that the amplitude of facilitation differs depending on the target neurons and the way of stimulation (Isope and Barbour, 2002; Sims and Hartell, 2005; Beierlein et al., 2007). Unexpectedly, this is not the case for GC $\rightarrow$ $\mathrm{BC}$ synapses, which exhibit synaptic depression. The difference $2.4 \mathrm{mV}$ (SR 95531).
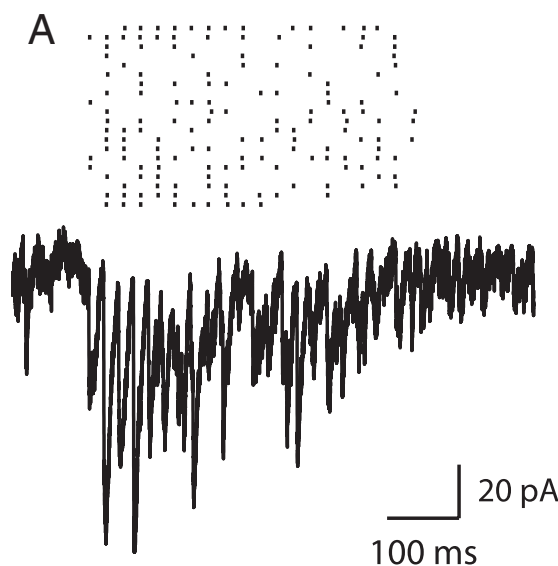

B

Figure 6. $\mathrm{SC} \rightarrow \mathrm{PC}$ connection with realistic firing pattern of $\mathrm{SC}$ under $50 \mathrm{~Hz} \mathrm{GC}$ stimulation. $A$, Firing patterns of an example $\mathrm{SC}$ in response to the $\mathrm{GC}$ stimulation at $50 \mathrm{~Hz}$ (Fig. 4B) was applied to stellate cells. Twenty different patterns were applied, and the resulting IPSCs from the PC were averaged (bottom trace). $\boldsymbol{B}$, The summary of normalized peak amplitudes from seven cells.

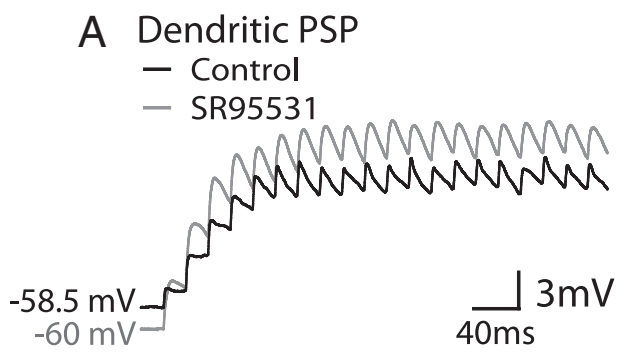

\section{B Subtracted IPSP}
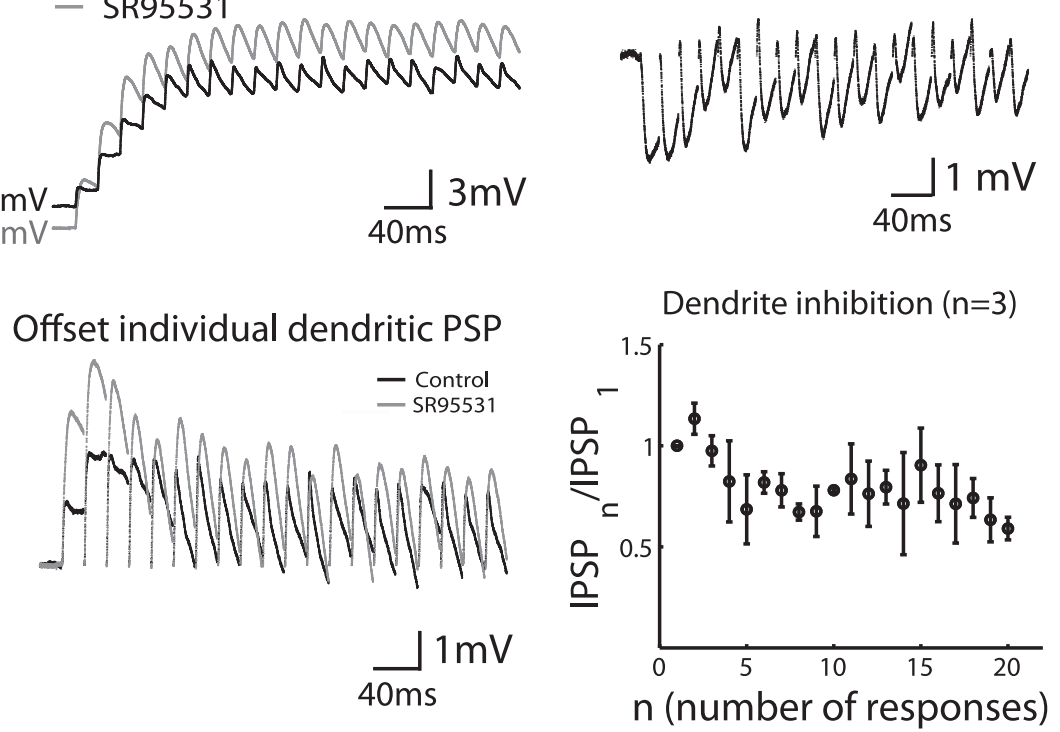

Figure 7. Persistent dendritic inhibitory potentials. A, PSPs were recorded from the dendritic patch pipette during a 50 $\mathrm{Hz}$ granule cell stimulation in both control (black) and in the presence of SR 95531 (gray) conditions (top). Each PSP was offset by the decay of its previous response to highlight the difference between the two conditions during each stimulation (bottom). B , Subtracting the two traces resulted in IPSPs that were blocked by SR 95531 (top). The peak value of each IPSP during the train was normalized to the first one. An average of three cells is plotted against the number of responses showing $80 \pm 4.7 \%$ steady-state depression. Resting membrane potential at the dendrite was $-60.5 \pm 1.5 \mathrm{mV}$ (control) and $-64.2 \pm 2.1 \mathrm{mV}$ (SR 95531). Resting membrane potential at the soma was $-59.5 \pm 0.7 \mathrm{mV}$ (control) and $-62.3 \pm$

between the facilitating PF synapses and depressing PF synapses may come from different synaptic locations on the granule cell axon (Sims and Hartell, 2005). Stellate cells are more innervated by the parallel fiber synapses, whereas basket cells are innervated by both the parallel fiber synapses and the ascending axon synapses. Furthermore, we have shown that Munc13-3 is important for short-term synaptic plasticity at GC $\rightarrow$ BC synapses. Because Munc13-3 is a presynaptic protein, presynaptic factors must play important roles for the synaptic depression at GC $\rightarrow$ BC synapses. Synaptic depression is normally achieved as a result of the initial high release probability (Zucker and Regehr, 2002), but the 


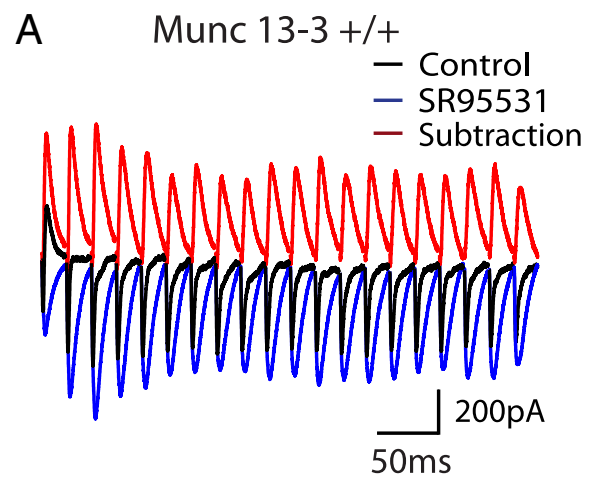

B Munc $13-3-/-$
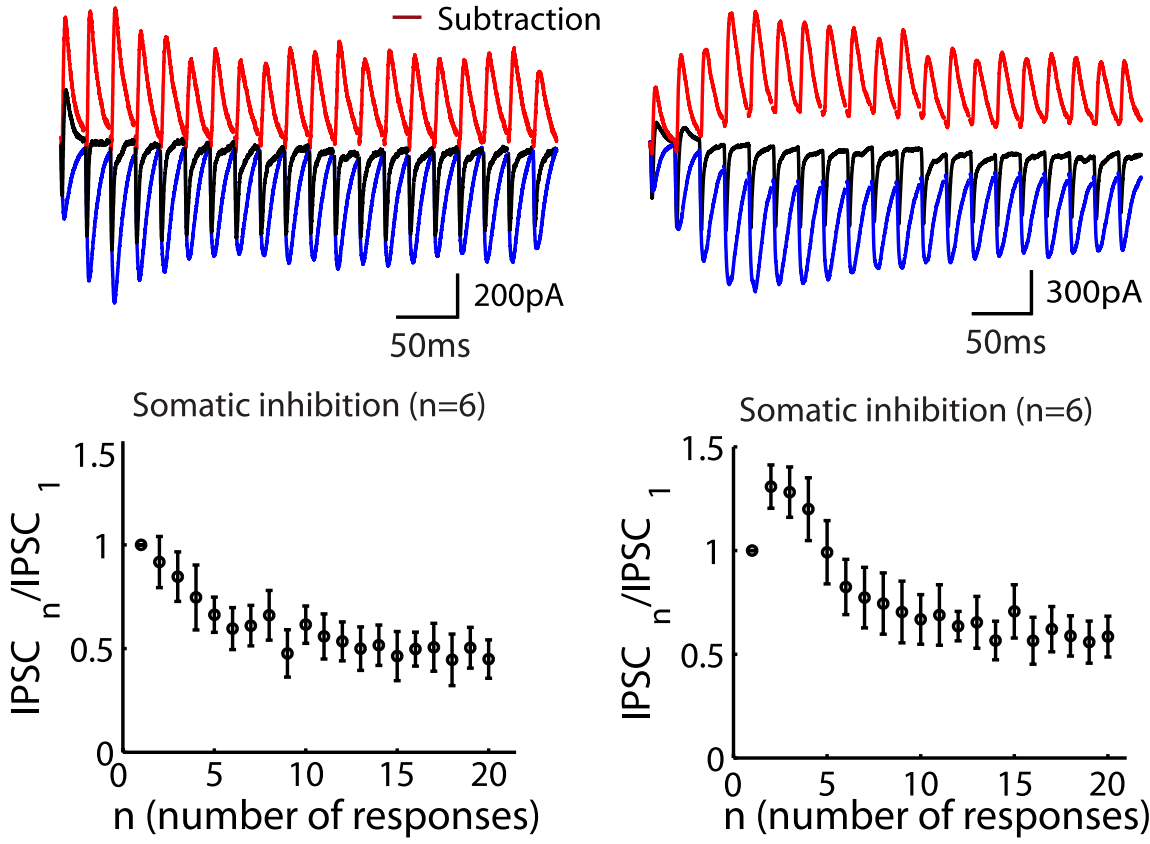

Figure 8. Phasic somatic inhibition. A, Somatic recorded PSCs from a PC under $50 \mathrm{~Hz}$ GC stimulations are shown in both control (black) and SR 95531 (blue) conditions (top). Subtracting the two traces gave an estimation of somatic IPSCS (red). Normalized IPSCs were averaged from six cells showing $48 \pm 1.5 \%$ steady-state depression. $\boldsymbol{B}$, The same experiment as in $\boldsymbol{A}$, but the data were obtained from Munc13-3 K0 mice.

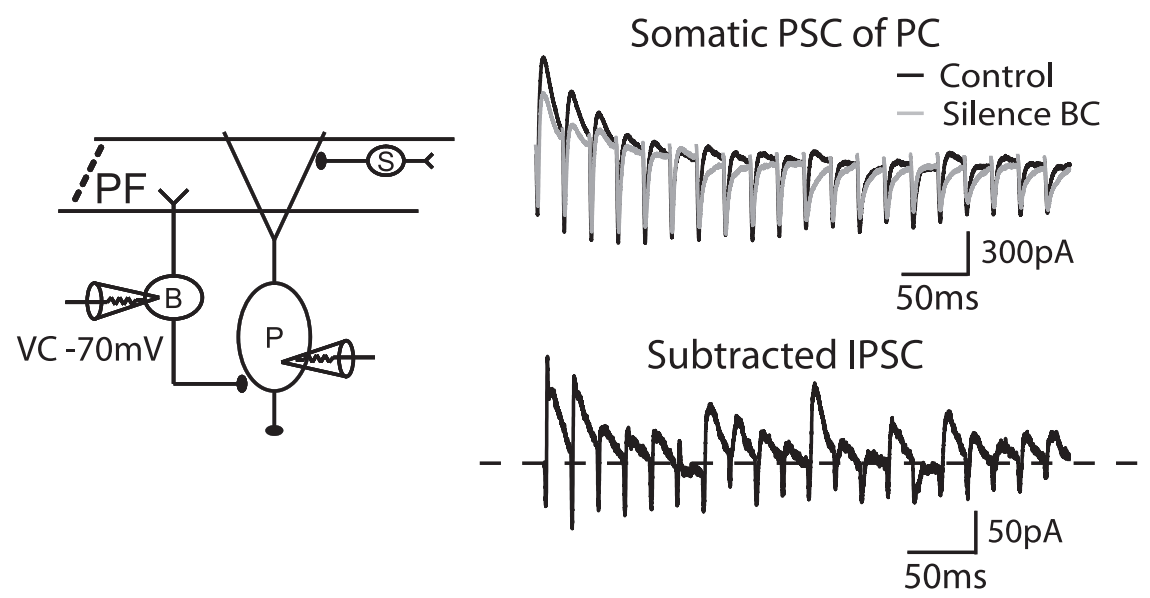

Figure 9. Silencing a single basket cell indicates phasic somatic inhibition. A diagram showing the experimental scheme of silencing one $B C$ by voltage clamping the $B C$ at $-70 \mathrm{mV}$ during a paired recording of $B C$ and $P C$ (left). Somatic $P S C$ recorded from the $\mathrm{PC}$ under $50 \mathrm{~Hz} \mathrm{GC}$ stimulations are shown in control condition (black, when the $\mathrm{BC}$ is under current-clamp mode) and in the condition when the $B C$ is silenced (gray). Subtracting the two traces shows the IPSCs delivered by one $B C$ onto the soma of the $P C$ (bottom panel, right).Zero level is indicated as a dotted line. An average of $40 \pm 5.8 \%$ steady-state depression was observed from four cells. B, Basket cell; P, Purkinje cell; S, Stellate cell.

precise mechanism as to how Munc13-3 affects the vesicle release probability remains to be tested.

\section{Subcellular compartment target determines the inhibitory synaptic strength}

Unlike neocortical (Beierlein et al., 2003) and hippocampal (Pouille and Scanziani, 2004; Hefft and Jonas, 2005) inhibitory circuits in which different types of interneurons are recruited, in the cerebellar FFI circuit, we showed that both types of molecular layer interneurons were fast-spiking neurons and their output synapses exhibited similar short-term dynamics. This supports the idea that BCs and SCs are similar types of interneurons that differ only in morphology and spatial distribution within the molecular layer (Sultan and Bower, 1998). Connections made by a BC and a SC onto the soma and dendrite of a PC have a sevenfold difference in synaptic strength. This difference is approximately twice as large as reported previously in the young rat (Vincent and Marty, 1996). However, it contrasts with the somatic and dendritic inhibition in the hippocampus (Miles et al., 1996) in which less than a twofold difference in synaptic strength was reported. One parallel fiber makes one or two synapses with a PC (Palay and Chan-Palay, 1974), and unitary granule cell $\rightarrow$ Purkinje cell connection is weak (Isope and Barbour, 2002). If the main function of the dendritic FFI is to balance the excitation, a comparable inhibitory synaptic strength with excitatory one will be the optimal solution concerning both function and energy consumption (Niven and Laughlin, 2008). As for somatic inhibition, it confronts strong PF inputs summed over the entire dendrites and climbing fiber inputs (Eccles et al., 1966c). A strong inhibitory synapse is recruited at the soma to balance strong excitatory inputs and inhibit the spike output of the Purkinje cell. We demonstrated that both BC and SC had broad dynamic range for spiking output (the feature of fast spiking neuron), within which the input synaptic dynamics can be precisely transformed to the dynamics of output firing rate. As a consequence, $\mathrm{PF}$ synapses are the main determinants for the recruitment of interneurons in the cerebellar FFI circuit. Furthermore, because BC and SC share similar output synaptic dynamics, both FFI pathways will be tightly regulated by the dynamics of excitatory inputs to the circuit, which is different from inhibitory circuits in other brain regions (Beierlein et al., 2003; Pouille and Scanziani, 2004) in which various types of interneurons show distinct forms of shortterm synaptic plasticity.
Functional implications

Inhibitory circuits are recruited in a frequency-dependent manner in both hippocampal FFI circuits (Mori et al., 2004; Klyachko and Stevens, 2006) and feedback circuits (Pouille and Scanziani, 2004). We reported here a similar phenomenon in cerebellum and further showed that the frequency-dependent recruitment depended on the targeted subcellular compartments (soma vs dendrite). Driven by high-frequency GC inputs (Chadderton et al., 2004; Jörntell and Ekerot, 2006), the somatic FFI circuit op- 
erates more effectively at the onset of a stimulation, whereas the dendritic FFI circuit can follow the activity of GCs. Note that difference in the magnitude of short-term synaptic plasticity between GC to BC and SC synapses was largest in the first 5 or 10 stimulations. Under physiological conditions, such a number of spikes were elicited by sensory stimulation (Chadderton et al., 2004). Another possible consequence of the depressing feature of the somatic inhibition is to detect synchronized activities of multiple BCs that innervate the same PC. In vivo, molecular layer interneurons fire spontaneously at low rates (Eccles et al., 1966a; Vincent and Marty, 1993; Häusser and Clark, 1997). Because of slow recovery from synaptic depression (Sakaba, 2008), each BC input may be already at the depressed state in the presence of spontaneous activities. Then, only when several BCs fire simultaneously, a substantial inhibition can inhibit the spike output of the PC. The BCs that innervate the same PC lie in the plane orthogonal to the PFs, and each PC is innervated by multiple BCs (Palay and ChanPalay, 1974). Therefore, each BC carries the information of a distinct group of GCs to the PC. Detecting the synchrony of multiple BCs may play an important role in integrating the spatial information over a wide range of GCs. As shown in Figure 7, the dendritic inhibition works as a scaling factor for the excitatory inputs and shortens the membrane time constant to rapidly terminate membrane depolarization. Its broad frequency response property may suggest an important functional consequence of balancing the dendritic depolarization to prevent the activation of voltage-gated active conductances such as $\mathrm{Ca}^{2+}$ spikes (Llinás et al., 1968; Miles et al., 1996; London and Häusser, 2005). In general, frequency tuning appears to be a major function for neuronal circuits, but its property differs among preparations (Beierlein et al., 2003; Lawrence and McBain, 2003; Mori et al., 2004; Pouille and Scanziani, 2004). The remaining task is to find out the relevance of the tuning property with the physiological task of the circuit in vivo.

\section{References}

Abbott LF, Regehr WG (2004) Synaptic computation. Nature 431:796-803. Atluri PP, Regehr WG (1996) Determinants of the time course of facilitation at the granule cell to Purkinje cell synapse. J Neurosci 16:5661-5671.

Augustin I, Betz A, Herrmann C, Jo T, Brose N (1999) Differential expression of two novel munc13 proteins in rat brain. Biochem J 337:363-371.

Augustin I, Korte S, Rickmann M, Kretzschmar HA, Südhof TC, Herms JW, Brose N (2001) The cerebellum-specific Munc13 isoform Munc13-3 regulates cerebellar synaptic transmission and motor learning in mice. J Neurosci 21:10-17.

Barbour B (1993) Synaptic currents evoked in Purkinje cells by stimulating individual granule cells. Neuron 11:759-769.

Basu J, Betz A, Brose N, Rosenmund C (2007) Munc13-1 C1 domain activation lowers the energy barrier for synaptic vesicle fusion. J Neurosci 27:1200-1210.

Beierlein M, Gibson JR, Connors BW (2003) Two dynamically distinct inhibitory networks in layer 4 of the neocortex. J Neurophysiol 90:2987-3000.

Beierlein M, Fioravante D, Regehr WG (2007) Differential expression of posttetanic potentiation and retrograde signaling mediate target-dependent short-term synaptic plasticity. Neuron 54:949-959.

Brunel N, Hakim V, Isope P, Nadal JP, Barbour B (2004) Optimal information storage and the distribution of synaptic weights: perceptron versus Purkinje cell. Neuron 43:745-757.

Caillard O, Moreno H, Schwaller B, Llano I, Celio MR, Marty A (2000) Role of the calcium-binding protein parvalbumin in short-term synaptic plasticity. Proc Natl Acad Sci U S A 97:13372-13377.

Chadderton P, Margrie TW, Häusser M (2004) Integration of quanta in cerebellar granule cells during sensory processing. Nature 428:856-860.
Dittman JS, Kreitzer AC, Regehr WG (2000) Interplay between facilitation, depression, and residual calcium at three presynaptic terminals. J Neurosci 20:1374-1385.

Eccles JC, Llinás R, Sasaki K (1966a) The inhibitory interneurons within the cerebellar cortex. Exp Brain Res 1:1-16.

Eccles JC, Llinás R, Sasaki K (1966b) Parallel fibre stimulation and the responses induced thereby in the Purkinje cells of the cerebellum. Exp Brain Res 1:17-39.

Eccles JC, Llinás R, Sasaki K (1966c) The excitatory synaptic action of climbing fibres on the Purkinje cells of the cerebellum. J Physiol 182:268-296.

Eccles JC, Llinás R, Sasaki K (1966d) The action of antidromic impulses on the cerebellar Purkinje cells. J Physiol 182:316-345.

Eccles JC, Ito M, Szenthagothai J (1967) The cerebellum as a neuronal machine. Heidelberg: Springer.

Häusser M, Clark BA (1997) Tonic synaptic inhibition modulates neuronal output pattern and spatiotemporal synaptic integration. Neuron 19:665678.

Hefft S, Jonas P (2005) Asynchronous gaba release generates long-lasting inhibition at a hippocampal interneuron-principal neuron synapse. Nat Neurosci 8:1319-1328.

Isope P, Barbour B (2002) Properties of unitary granule cell $\rightarrow$ Purkinje cell synapses in adult rat cerebellar slices. J Neurosci 22:9668-9678.

Jörntell H, Ekerot CF (2006) Properties of somatosensory synaptic integration in cerebellar granule cells in vivo. J Neurosci 26:11786-11797.

Klyachko V, Stevens C (2006) Excitatory and feed-forward inhibitory hippocampal synapses work synergistically as an adaptive filter of natural spike trains. PLoS Biol 4:e207.

Konnerth A, Llano I, Armstrong CM (1990) Synaptic currents in cerebellar Purkinje cells. Proc Natl Acad Sci U S A 87:2662-2665.

Kosaka T, Kosaka K, Nakayama T, Hunziker W, Heizmann CW (1993) Axons and axon terminals of cerebellar Purkinje cells and basket cells have higher levels of parvalbumin immunoreactivity than somata and dendrites: quantitative analysis by immunogold labeling. Exp Brain Res 93:483-491.

Lawrence JJ, McBain CJ (2003) Interneuron diversity series: containing the detonation-feedforward inhibition in the ca3 hippocampus. Trends Neurosci 26:631-640.

Llinás R, Nicholson C, Freeman JA, Hillman DE (1968) Dendritic spikes and their inhibition in alligator Purkinje cells. Science 160:1132-1135.

London M, Häusser M (2005) Dendritic computation. Annu Rev Neurosci 28:503-532.

Marcaggi P, Attwell D (2005) Endocannabinoid signaling depends on the spatial pattern of synapse activation. Nat Neurosci 8:776-781.

Markram H, Toledo-Rodriguez M, Wang Y, Gupta A, Silberberg G, Wu C (2004) Interneurons of the neocortical inhibitory system. Nat Rev Neurosci 5:793-807.

Miles R, Tóth K, Gulyás AI, Hájos N, Freund TF (1996) Differences between somatic and dendritic inhibition in the hippocampus. Neuron $16: 815-823$.

Mittmann W, Häusser M (2007) Linking synaptic plasticity and spike output at excitatory and inhibitory synapses onto cerebellar Purkinje cells. J Neurosci 27:5559-5570.

Mittmann W, Koch U, Häusser M (2005) Feed-forward inhibition shapes the spike output of cerebellar Purkinje cells. J Physiol 563:369-378.

Miyashita Y, Nagao S (1984) Contribution of cerebellar intracortical inhibition to Purkinje cell response during vestibulo-ocular reflex of alert rabbits. J Physiol 351:251-262.

Mori M, Abegg MH, Gähwiler BH, Gerber U (2004) A frequencydependent switch from inhibition to excitation in a hippocampal unitary circuit. Nature 431:453-456.

Niven JE, Laughlin SB (2008) Energy limitation as a selective pressure on the evolution of sensory systems. J Exp Biol 211:1792-1804.

Palay SL, Chan-Palay V (1974) Cerebellar cortex: cytology and organization. Berlin: Springer.

Pouille F, Scanziani M (2004) Routing of spike series by dynamic circuits in the hippocampus. Nature 429:717-723.

Reyes A, Lujan R, Rozov A, Burnashev N, Somogyi P, Sakmann B (1998) Target-cell-specific facilitation and depression in neocortical circuits. Nat Neurosci 1:279-285.

Sakaba T (2008) Two $\mathrm{Ca}^{2+}$-dependent steps controlling synaptic vesicle 
fusion and replenishment at the cerebellar basket cell terminal. Neuron 57:406-419.

Silver RA, Momiyama A, Cull-Candy SG (1998) Locus of frequencydependent depression identified with multiple-probability fluctuation analysis at rat climbing fibre-Purkinje cell synapses. J Physiol 510:881902.

Sims RE, Hartell NA (2005) Differences in transmission properties and susceptibility to long-term depression reveal functional specialization of ascending axon and parallel fiber synapses to Purkinje cells. J Neurosci 25:3246-3257.

Somogyi P, Klausberger T (2005) Defined types of cortical interneurone structure space and spike timing in the hippocampus. J Physiol 562:9-26.

Sultan F, Bower JM (1998) Quantitative Golgi study of the rat cerebellar molecular layer interneurons using principal component analysis. J Comp Neurol 393:353-373.

Vincent P, Marty A (1993) Neighboring cerebellar Purkinje cells communicate via retrograde inhibition of common presynaptic interneurons. Neuron 11:885-893.

Vincent P, Marty A (1996) Fluctuations of inhibitory postsynaptic currents in Purkinje cells from rat cerebellar slices. J Physiol 494:183-199.

Wulff $\mathrm{P}$, Schonewille M, Renzi M, Viltono L, Sassoè-Pognetto M, Badura A, Gao Z, Hoebeek FE, van Dorp S, Wisden W, Farrant M, De Zeeuw CI (2009) Synaptic inhibition of Purkinje cells mediates consolidation of vestibulo-cerebellar motor learning. Nat Neurosci 12:1042-1049.

Zucker RS, Regehr WG (2002) Short-term synaptic plasticity. Annu Rev Physiol 64:355-405. 